\title{
Nonconcave entropies in multifractals and the thermodynamic formalism
}

\author{
Hugo Touchette ${ }^{*}$ and Christian Beck $\mathrm{H}^{-1}$ \\ School of Mathematical Sciences, Queen Mary, University of London, London E1 4NS, UK
}

(Dated: August 13, 2018)

\begin{abstract}
We discuss a subtlety involved in the calculation of multifractal spectra when these are expressed as Legendre-Fenchel transforms of functions analogous to free energy functions. We show that the Legendre-Fenchel transform of a free energy function yields the correct multifractal spectrum only when the latter is wholly concave. If the spectrum has no definite concavity, then the transform yields the concave envelope of the spectrum rather than the spectrum itself. Some mathematical and physical examples are given to illustrate this result, which lies at the root of the nonequivalence of the microcanonical and canonical ensembles. On a more positive note, we also show that the impossibility of expressing nonconcave multifractal spectra through Legendre-Fenchel transforms of free energies can be circumvented with the help of a generalized free energy function, which relates to a recently introduced generalized canonical ensemble. Analogies with the calculation of rate functions in large deviation theory are finally discussed.
\end{abstract}

PACS numbers: 05.45.Df, 64.60.Ak, 65.40.Gr

\section{INTRODUCTION}

Invariant measures generated by nonlinear and complex dynamical systems often show striking scaling and self-similar features that are reminiscent of fractals. However, contrary to ordinary fractals, whose geometric structure is characterized by a single number (the fractal or Hausdorff dimension [1]), the scaling and self-similar properties of measures are usually not captured by a single dimension, say $\alpha$, but by an infinite set of fractal or singularity dimensions that defines the so-called spectrum of singularities $f(\alpha)$, also known as the multifractal spectrum [2, 3]. The word "multifractal" has been coined [4] in this context precisely to suggest that a measure having multiscaling properties can be pictured abstractly as a superposition of many "pure" fractals, each having a dimension $\alpha$ and a corresponding "weight" $f(\alpha)$ in the superposition.

To be more specific, consider a measure $\mu$ defined on a $d$-dimensional space $X$. Generalizing the approach followed in fractal geometry, we proceed to partition or "coarse-grain" the space $X$ in small boxes of equal size $\varepsilon$ and volume $\varepsilon^{d}$. The measure contained in each box is

$$
p_{\varepsilon, i}=\int_{i^{\text {th }} \text { box }} d \mu(x),
$$

and from this quantity, a local fractal dimension $\alpha_{i}$, also called a crowding index, is defined by using the fact that $p_{\varepsilon, i}$ is expected to scale as $p_{\varepsilon, i} \sim \varepsilon^{\alpha_{i}}$ in the limit where the boxes' size $\varepsilon$ goes to zero. Now, to account for the fact that $\alpha_{i}$ is not constant over the partition but varies in general from one box to another, we count the number $n_{\varepsilon}(\alpha)$ of boxes in the partition whose local dimension is equal to $\alpha$. From $n_{\varepsilon}(\alpha)$, the multifractal spectrum $f(\alpha)$ is then simply defined through another scaling relationship, namely $n_{\varepsilon}(\alpha) \sim \varepsilon^{-f(\alpha)}$ as $\varepsilon \rightarrow 0$.

The multifractal spectrum $f(\alpha)$ is not a quantity which is easily calculated analytically or numerically, since it requires the enumeration of all the boxes in the partition of $X$ having a crowding index $\alpha$ lying in some interval $[\alpha, \alpha+\Delta \alpha]$. A more manageable quantity which can be related to $f(\alpha)$ is the so-called free energy function $\tau(q)$ defined by the scaling relationship $Z_{\varepsilon}(q) \sim \varepsilon^{\tau(q)}, \varepsilon \rightarrow 0$, where

$$
Z_{\varepsilon}(q)=\sum_{i} p_{\varepsilon, i}^{q} \sim \sum_{i} \varepsilon^{-q \alpha_{i}}
$$

is the partition function associated with the partition $X$ of $\mu$ (the sum above runs over all the boxes of the partition with $p_{\varepsilon, i} \neq 0$ since $q$ can be negative). The calculation of $\tau(q)$ parallels the calculation of free energies in statistical mechanics in that, if $f(\alpha)$ is known, then $\tau(q)$ can be calculated as the Legendre-Fenchel (LF) transform of $f(\alpha)$ [2]; in symbols,

$$
\tau(q)=\inf _{\alpha \in \mathbb{R}}\{q \alpha-f(\alpha)\} .
$$

The result that we shall study in this paper is the inverse result, namely that if $\tau(q)$ is known, then $f(\alpha)$ can be calculated from $\tau(q)$ by taking the LF transform of the latter function; in symbols,

$$
f(\alpha)=\inf _{q \in \mathbb{R}}\{q \alpha-\tau(q)\} .
$$

This result first appeared in Refs. [4, [5], and has been used extensively since then to calculate the multifractal spectrum of many phenomena, including turbulence [6, 77, 8, 9, 10, 11], geophysical processes, such as cloud formation and rain precipitations [12, 13, 14], and fluctuations in financial time series $[3,15]$, among many others [16]. Unfortunately, there is one aspect of Eq.(44) which is often overlooked when deriving it and applying it, namely that it can only produce concave multifractal spectra, since LF transforms can only yield concave functions. This basic property of LF transforms does not affect, as such, the calculation of $\tau(q)$ from $f(\alpha)$ because it can be proved that $\tau(q)$ is an always concave function of $q$. For calculating the multifractal spectrum, however, 
(a)

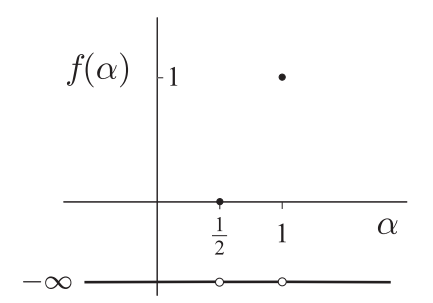

(b)

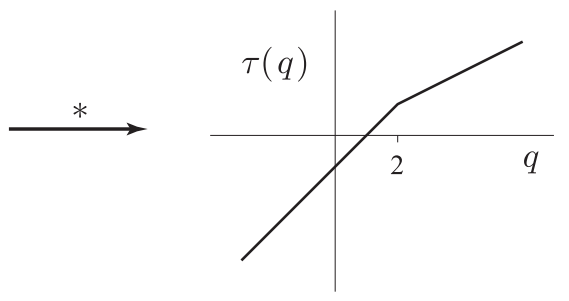

(c)

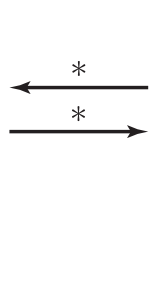

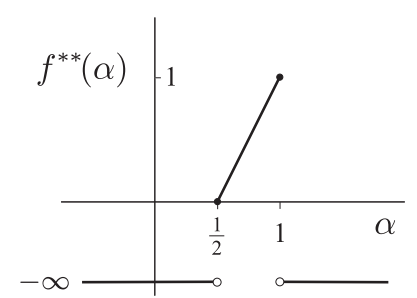

FIG. 1: (a) Multifractal spectrum $f(\alpha)$ for the invariant density of the Ulam map. (b) Corresponding free energy function $\tau(q)$. (c) Legendre-Fenchel transform of $\tau(q)$.

there is a problem because $f(\alpha)$ need not be concave, which means that $f(\alpha)$ cannot always be calculated as the LF transform of $\tau(q)$.

Our goal here is to illustrate these observations with a number of basic examples, and to state the precise conditions, based on convex analysis, that ensure that $f(\alpha)$ can be calculated as the LF transform of $\tau(q)$. These conditions will be discussed in the context of four physicallyrelevant multifractal models: one related to turbulence, another related to diffusion-limited aggregates, and two others related to chaotic systems. In an attempt to offer a workable solution to the problem of calculating nonconcave multifractal spectra, we shall also study a recently introduced generalized canonical ensemble to show that nonconcave spectra can be obtained from a modified version of the LF transform. This part will actually provide an explicit calculation of a nonconcave spectrum $f(\alpha)$ which uses this modified LF transform. We shall comment finally, in the concluding section of the paper, on analogies between nonconcave multifractal spectra, nonconcave entropies in statistical mechanics, and large deviation theory.

\section{TWO SIMPLE EXAMPLES}

We begin by considering two explicit examples of measures whose multifractal spectra are not given by LF transforms of their free energy functions. The first example was previously discussed in Ref. [17] (see also Ref. [2]), and will serve here as a starting point to our discussion of the validity of the LF transform of (4). The measure or, rather, the density in this case that we consider is given by

$$
\rho(x)=\frac{1}{\pi \sqrt{1-x^{2}}},
$$

where $x \in[-1,1]$. This density arises as the invariant density of the Ulam map and the Tchebyscheff maps. Applying a partition of size $\varepsilon$ on the interval $[-1,1]$, it can be seen that the two boxes of the partition located near the boundary points $x= \pm 1$ have measure $p_{\varepsilon, i}=$ $\varepsilon \rho(x) \sim \varepsilon^{1 / 2}$, so that $f(\alpha)=0$ at $\alpha=1 / 2$. All the other boxes have measure $p_{\varepsilon, i} \sim \varepsilon$, so that $f(\alpha)=1$ at $\alpha=1$, as there are approximately $n_{\varepsilon}(\alpha) \sim \varepsilon^{-1}$ of these boxes. Combining the two results, and setting $n_{\varepsilon}(\alpha)=0$ for $\alpha \notin\{1 / 2,1\}$, we obtain

$$
f(\alpha)= \begin{cases}0 & \alpha=1 / 2 \\ 1 & \alpha=1 \\ -\infty & \text { otherwise }\end{cases}
$$

This spectrum is shown in Fig. 1(a).

At this point, we go on to prove that $f(\alpha)$ cannot be expressed as the LF transform of $\tau(q)$ by direct calculation. Starting from the asymptotic $(\varepsilon \rightarrow 0)$ expression of the partition function

$$
Z_{\varepsilon}(q)=\sum_{i} p_{\varepsilon, i}^{q} \sim \varepsilon^{q / 2}+\varepsilon^{-1} \varepsilon^{q},
$$

we first find

$$
\tau(q)=\min \{q-1, q / 2\}= \begin{cases}q / 2 & q>2 \\ q-1 & q \leq 2\end{cases}
$$

Then keeping track of the two separate regions $q>2$ and $q \leq 2$, we find

$$
\begin{aligned}
\inf _{q \in \mathbb{R}}\{q \alpha-\tau(q)\} & =\inf _{q \in \mathbb{R}}\left\{\begin{array}{ll}
q\left(\alpha-\frac{1}{2}\right) & q>2 \\
q(\alpha-1)+1 & q \leq 2
\end{array}\right\} \\
& = \begin{cases}2 \alpha-1 & \alpha \in[1 / 2,1] \\
-\infty & \text { otherwise. }\end{cases}
\end{aligned}
$$

Comparing this result with Eq.(6), we see that $f(\alpha)$ corresponds to the LF transform of $\tau(q)$ for $\alpha \notin(1 / 2,1)$ only; see Figs. 1(a) and 1(c). For $\alpha \in(1 / 2,1)$, the LF transform of $\tau(q)$ is finite, while the true spectrum $f(\alpha)$ is formally equal to $-\infty$, as there is no box in the partition of $\rho(x)$ with local exponent in the range $(1 / 2,1)$.

This example can be generalized to illustrate another problem when trying to obtain $f(\alpha)$ from $\tau(q)$. Consider a dynamical system in $d$-dimensions whose invariant density is everywhere finite, so that $p_{i, \varepsilon} \sim \varepsilon^{d}$, except at a finite number $k$ of singular points where $p_{i, \varepsilon} \sim \varepsilon^{\alpha_{i}}=\varepsilon^{d \xi_{i}}$ with $\xi_{1}, \xi_{2}, \ldots, \xi_{k}<1$. The partition function for this density is

$$
Z_{\varepsilon}(q)=\sum_{i} p_{i}^{q} \sim \varepsilon^{-d} \varepsilon^{d q}+\varepsilon^{d \xi_{1} q}+\varepsilon^{d \xi_{2} q}+\cdots+\varepsilon^{d \xi_{k} q},
$$


so that

$$
\tau(q)=\min \left\{(q-1) d, d \xi_{1} q, d \xi_{2} q, \ldots, d \xi_{k} q\right\} .
$$

The minimum can be calculated explicitly and yields

$$
\tau(q)= \begin{cases}d(q-1) & q \leq\left(1-\xi^{*}\right)^{-1} \\ d \xi^{*} q & q \geq\left(1-\xi^{*}\right)^{-1}\end{cases}
$$

where $\xi^{*}=\min _{i} \xi_{i}$. We see here that the function $\tau(q)$ "overlooks" all the singularities $\xi_{i}$, except for the smallest one. Therefore, any perturbation of the singularities $\xi_{i}$ that keeps $\xi^{*}$ invariant will change $f(\alpha)$ but not $\tau(q)$, which implies that the mapping of $\tau(q)$ to $f(\alpha)$ must be indeterminate as there is an infinite number of spectra associated with the same free energy. Physically, this also implies that $\tau(q)$ does not offer the most complete description of the dynamical system, since this function overlooks, as we said, all but one singularity. To really obtain a complete picture of all the singularities of the system, one must resort to calculate $f(\alpha)$ and not just $\tau(q)$.

\section{THEORY OF LF TRANSFORMS}

The results of the two previous examples are very simple and show at once that $f(\alpha)$ cannot in general be expressed as the LF transform of $\tau(q)$, contrary to what is claimed in most if not all references on the subject. The problem, as was mentioned, is that LF transforms can only yield concave functions, which means that these transforms cannot be used to calculate nonconcave multifractal spectra, including those of the two examples considered before. To make this observation more rigorous, we introduce in this section a few concepts and results of convex analysis, beginning with the concept of supporting lines. (All the definitions and theorems discussed here can be found in Ref. [18]; see also Chapter VI of Ref. [19] and Appendix A of Ref. 20].)

Definition 1. A function $f: \mathbb{R} \rightarrow \mathbb{R}$ admits a supporting line at $\alpha$ if there exists a constant $\eta$ such that

$$
f(\beta) \leq f(\alpha)+\eta(\beta-\alpha)
$$

for all $\beta \in \mathbb{R}$.

This definition means graphically that we can draw a line on top of the graph of $f(\alpha)$ that does not go under that graph (see Fig. 2); hence the word "supporting." With this picture in mind, it is easily seen that, if $f$ admits a supporting line at $\alpha$ and is differentiable at $\alpha$, then the slope $\eta$ of the supporting line must be such that $f^{\prime}(\alpha)=\eta$.

The importance of supporting lines comes from their association with LF transforms, and from the fact, more precisely, that they determine whether such transforms are involutive, that is, whether they are their own inverse. In the context of $f(\alpha)$ and $\tau(q)$, this means precisely the

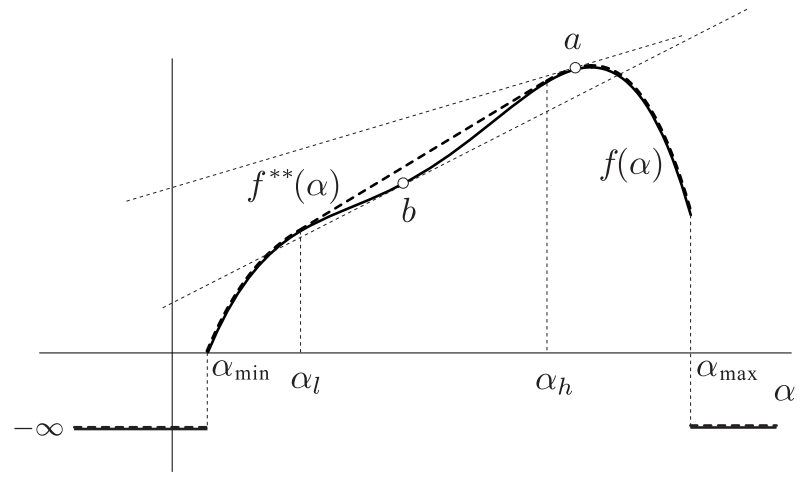

FIG. 2: (a) A generic nonconcave multifractal spectrum $f(\alpha)$ (full line) together with its concave envelope $f^{* *}(\alpha)$ (dashed line). The two functions coincide outside the open interval $\left(\alpha_{l}, \alpha_{h}\right)$. The point $a$ of the multifractal spectrum admits a supporting line (concave point), while the point $b$ does not (nonconcave point).

following. First, recall that $\tau(q)$ can always be expressed as the LF transform of $f(\alpha)$, so Eq.(3) is always valid independently of the shape of $f(\alpha)$. This follows essentially from the fact that $\tau(q)$ is an always concave function of $q$ [2], a fact that can be proved using Hölder's inequality. The inverse transform shown in (4), however, is not generally valid, and this is where supporting lines become important, as expressed in the next theorem.

Theorem 2. If $f$ admits a supporting line at $\alpha$, then $f$ at $\alpha$ can expressed as the LF transform of $\tau(q)$ as in Eq.(44). In this case, we say that $f$ is concave at $\alpha$. On the other hand, if $f$ does not admit a supporting line at $\alpha$, then $f$ at $\alpha$ does not equal the LF transform of $\tau(q)$. In this case, we say that $f$ is nonconcave at $\alpha$.

The two complementary results expressed in the theorem above are usually rephrased in convex analysis by defining the function

$$
f^{* *}(\alpha)=\inf _{q \in \mathbb{R}}\{q \alpha-\tau(q)\} .
$$

In terms of $f^{* *}(\alpha)$, we then have the following result.

Theorem 3. $f(\alpha)=f^{* *}(\alpha)$ if and only if $f$ admits a supporting line at $\alpha$.

For the remaining, it is useful to note that $f^{* *}(\alpha)$ corresponds in general to the smallest concave function satisfying $f(\alpha) \leq f^{* *}(\alpha)$ for all $\alpha \in \mathbb{R}$. For this reason, $f^{* *}(\alpha)$ is called the concave envelope or concave hull of $f(\alpha)$. This implies, in particular, that if $f(\alpha)$ admits no supporting lines over some open interval, say $\left(\alpha_{l}, \alpha_{h}\right)$ as in Fig. 2, then $f^{* *}(\alpha)$ must be affine over that interval, by which we mean that $f^{* *}(\alpha)$ has a constant slope over that interval. This last property, which is related to the Maxwell construction [21, 22], is illustrated in Fig. 2.

All of the properties of $f(\alpha)$ and $f^{* *}(\alpha)$ in relation to LF transforms can be verified for the two examples considered previously. In the case of the invariant density 
of the Ulam map, for example, the concave hull of $f(\alpha)$ is the function displayed in (9); it is obviously such that $f(\alpha) \leq f^{* *}(\alpha)$ and is concave contrary to $f(\alpha)$. Moreover, it is easily verified from Fig. 1 that the two points $\alpha=1 / 2$ and $\alpha=1$ admit a supporting line, which explains why $f(\alpha)=f^{* *}(\alpha)$ there. These two points admit in fact an infinite number of supporting lines. For the point $\alpha=1 / 2$, for example, all lines attached to $(1 / 2,0)$ with slope in the interval $[2, \infty)$ are supporting in the sense of (13). For $\alpha=1$, the supporting lines have slopes in the interval $(-\infty, 2]$.

We can go further in our analysis of $f(\alpha)$ and $\tau(q)$ by calling attention to the fact that

$$
\tau(q)=\inf _{\alpha \in \mathbb{R}}\left\{q \alpha-f^{* *}(\alpha)\right\} .
$$

Therefore, $\tau(q)$ is not only the LF transform of $f(\alpha)$, as stated in Eq.(3), but also the LF transform of $f^{* *}(\alpha)$. This result is general: it holds for any function $f(\alpha)$ and its concave envelope $f^{* *}(\alpha)$ defined as in Eq.(14) as the double LF transform of $f(\alpha)$ or, more compactly, as

$$
f^{* *}=\tau^{*}=\left(f^{*}\right)^{*},
$$

where the star stands for the LF transformation. To summarize, we then have $\tau=f^{*}, \tau^{*}=\left(f^{*}\right)^{*}=f^{* *}$ and $\left(\tau^{*}\right)^{*}=\left(f^{* *}\right)^{*}=f^{*}=\tau$. This chain of equalities can be expressed in a more transparent way using the following diagram:

$$
f(\alpha) \stackrel{*}{\rightarrow} \tau(q) \stackrel{*}{\rightleftharpoons} f^{* *}(\alpha),
$$

which makes obvious the fact that there may be more than one spectrum related the same free energy. In fact, all $f(\alpha)$ having the same concave envelope lead to the same $\tau(q)$, as can be verified in the second example considered before. Finally, note that the chain of equalities reduces to $\tau=f^{*}$ and $\tau^{*}=f$, or equivalently to

$$
\tau(q) \stackrel{*}{\rightleftharpoons} f(\alpha),
$$

when $f(\alpha)=f^{* *}(\alpha)$ for all $\alpha \in \mathbb{R}$, that is, when $f(\alpha)$ is everywhere concave.

Having listed all the relationships that exist between $f(\alpha), \tau(q)$ and $f^{* *}(\alpha)$, we can now fully address the main issue of this paper, which is to determine when $f(\alpha)$ can safely and completely be calculated as the LF transform of $\tau(q)$. From the chain of equalities and diagrams shown above, this amounts to determine when the LF transform is involutive; that is to say, under which conditions does the diagram (17) reduce to the diagram of (18)?

A first obvious answer to this question is given by recalling what we have just mentioned about the diagram of (18), namely that if $f(\alpha)$ is everywhere concave, then the multifractal spectra $f(\alpha)$ can completely be calculated as the LF transform of the free energy function $\tau(q)$. As such, this answer is complete but not very practical because it is based on $f(\alpha)$ and so presupposes that we know $f(\alpha)$. A more useful criterion can be stated from

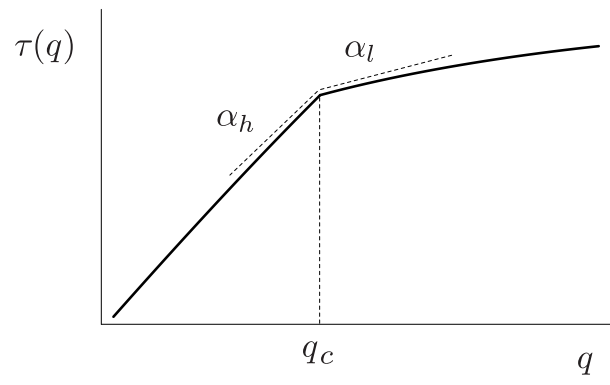

FIG. 3: Free energy function $\tau(q)$ associated with the multifractal spectrum $f(\alpha)$ shown in Fig. 2. The LF transform of the concave envelope $f^{* *}(\alpha)$ of $f(\alpha)$ yields the same free energy function.

the point of view of $\tau(q)$ alone by using a result of convex analysis connecting nonconcave or affine regions of $f(\alpha)$ with nondifferentiable points of $\tau(q)$. This result is stated next without a proof; see [18] for more details (see especially Theorems 23.5 and 26.3 ).

Theorem 4. Suppose that $f(\alpha)$ is nonconcave over some open interval $\left(\alpha_{l}, \alpha_{h}\right)$ (Fig. 2) or that $f(\alpha)$ is concave but affine over $\left(\alpha_{l}, \alpha_{h}\right)$. Then $\tau(q)$ is nondifferentiable at some critical value $q_{c}$ corresponding to the slope of $f^{* *}(\alpha)$ over the interval $\left(\alpha_{l}, \alpha_{h}\right)$. Moreover, the left- and rightderivatives of $\tau(q)$ at $q_{c}$ equal $\alpha_{h}$ and $\alpha_{l}$, respectively (Fig. 3).

From this result, we arrive at our criterion by taking the contrapositive: if $\tau(q)$ is everywhere differentiable, then $f(\alpha)$ is concave everywhere with no affine parts. Thus, from the point of view of $\tau(q), f(\alpha)$ can completely be calculated as the LF transform of $\tau(q)$ if the latter function is everywhere differentiable. Taking the view that nondifferentiable points of $\tau(q)$ represent first-order phase transitions for multifractals [23, 24, 25, 26, 27], this is equivalent to saying that $f(\alpha)$ can completely be calculated as the LF transform of $\tau(q)$ in the absence of first-order phase transitions. If there is a first-order phase transition, then either $f(\alpha)$ is nonconcave somewhere, in which case $f \neq \tau^{*}$, or else $f(\alpha)$ is affine somewhere, in which case $f=\tau^{*}$. Unfortunately - and this is an important point - there is no way to distinguish the two cases from the sole knowledge of $\tau(q)$ (see Figs. 2 and 3). Thus, if $\tau(q)$ has one or more nondifferentiable points and if there is no reason to think that $f(\alpha)$ is concave, then one must resort to calculate $f(\alpha)$ by means which do not rely on $\tau(q)$.

\section{APPLICATIONS}

We now revisit some examples of multifractal models that have been discussed in the physics literature, and point out where and how our results of the previous section apply. 


\section{A. Multifractal turbulence models}

One of the first field of study for which multifractal models have been developed is fully developed hydrodynamic turbulence [4, 7]. The basis of these models is that, in the turbulent flow, velocity increments $\delta v(l)=|v(x+l)-v(x)|$ at a given distance $l$ scale with local exponents $h$, which are distributed on a fractal set with fractal dimension $D(h)$. This notation is taken from Benzi et al. 7], and can be translated to our notation using the following identifications:

$$
\begin{aligned}
h & =\alpha \\
l & =\varepsilon \\
3-D(h) & =-f(\alpha) \\
p & =q \\
\zeta_{p} & =\tau(q) .
\end{aligned}
$$

Here $\zeta_{p}$ denote the scaling exponents of moments of velocity increments in the inertial range,

$$
\left\langle(\delta v)^{p}\right\rangle \sim l^{\zeta_{p}}
$$

In multifractal turbulence models, the probability to observe a local exponent $h$ is given by

$$
P_{l}(h) \sim l^{3-D(h)},
$$

which is equivalent to our notation $n_{\varepsilon}(\alpha) \sim \varepsilon^{-f(\alpha)}$. Moreover, for the scaling exponents $p$ one has

$$
\zeta_{p}=\min _{h}\{h p+3-D(h)\},
$$

which is equivalent to the LF transform of Eq.(3). In practice, one extracts $D(h)$ from the scaling exponents $\zeta_{p}$, which can be measured in experiments. In our notation, this means that one determines $f(\alpha)$ by the LF transform of the experimentally measured $\tau(q)$.

In view of all the results derived before, we can notice that multifractal turbulence models in their current form can only deal with the convex hull of the spectrum of singularities. The true spectrum $D(h)$ of a turbulent flow is fully determined by the underlying dynamics, i.e., the Navier-Stokes equation, and there is a priori no reason to think that this spectrum should be a concave function of $h$. Our arguments of the previous section now allow for an experimental check of the concavity of $D(h)$ : if the experimentally-measured $\zeta_{p}$ is differentiable within the precision allowed by the experiment, then $D(h)$ is concave and therefore given by the LF transform of $\zeta_{p}$. If one observes that $\zeta_{p}$ is nondifferentiable, then either $D(h)$ is nonconcave or else is affine. Both cases are consistent with the fact that $\zeta_{p}$ is nondifferentiable, but there is no way to tell from $\zeta_{p}$ which one of the two spectra is the actual one.

The exponents $\zeta_{p}$ have been measured in many experiments; see, e.g., Refs. [8, 9]. Within the experimental uncertainties, they are usually described by a smooth function of $p$, although one cannot fully exclude the existence of phase transitions. The data found in Refs. 8, 9], for example, show a relatively strong change of slope near $p=3$. If the existence of such transitions were confirmed (e.g., via the study of theoretical models of turbulence), then one would have to check that the underlying spectrum $D(h)$ is either nonconcave or concave but affine somewhere. If $D(h)$ is affine (see, e.g., Ref. [28]), then that spectrum is correctly given by the LF transform of $\zeta_{p}$. If $D(h)$ is nonconcave, then that spectrum is not fully given by the LF transform of $\zeta_{p}$. In this case, $\zeta_{p}$ cannot provide a complete description of the turbulent flow, since all the local exponents $h$ in the nonconcave region of $D(h)$ are "overlooked," in the spirit of the two simple examples discussed before, by $\zeta_{p}$. In short, these exponents are described by $D(h)$ but not by $\zeta_{p}$.

\section{B. Diffusion-limited aggregates}

For the second example, we consider multifractals as generated by diffusion-limited aggregates (DLA) [29, 30, 31]. Jensen et al. 31] provide convincing evidence that the function $\tau(q)$ calculated for the harmonic measure of their DLA cluster exhibits a first-order phase transition at $q=-0.23 \pm 0.05$ (see their Fig. 3). From this result, all that can be said about their $f(\alpha)$ spectrum obtained by LF-transforming $\tau(q)$ (shown in their Fig. 4) is that it is the concave hull $f^{* *}(\alpha)$ of the true $f(\alpha)$ spectrum. Note that the spectrum displayed in Fig. 4 of Ref. 31. does show an affine part, so it is consistent with the fact that $\tau(q)$ has a nondifferentiable point. However, there is a priori no reason why DLA clusters should possess a concave spectrum of singularities, so that the part where $f(\alpha)$ is seen to be affine could just as well be nonconcave. Therefore, at this point we may conclude that the true $f(\alpha)$ spectrum of the DLA cluster studied by Jensen et $a l$. is as yet unknown.

It should be remarked that, although Jensen et al. provide evidence to the effect that $\tau(q)$ possesses a nondifferentiable point, the $\tau(q)$ which is calculated in practice is actually always analytic if one deals with finite-size DLA clusters. The spectrum $f^{* *}(\alpha)$ which is calculated from $\tau(q)$ is, in this case, necessarily concave and has no affine parts. The nondifferentiable point of $\tau(q)$ and the concomitant affine part of $f^{* *}(\alpha)$ appear, formally speaking, only in the "thermodynamic" limit of infinitely large clusters.

\section{Chaotic systems}

Multifractal spectra have been calculated for many examples of chaotic dynamical systems, including the Hénon map [32] (see also Ref. 33]), and the driven damped pendulum [34]. The particularity of these two examples is that they seem to give rise to first-order phase transitions, which are also referred to as $q$-phase tran- 
sitions. Accordingly, the question arises as to whether these phase transitions emerge out of a nonconcave $f(\alpha)$ or an affine $f(\alpha)$.

The question, as it stands, is not resolved in the papers that treat these examples because they assume that $f(\alpha)$ is always the LF transform of $\tau(q)$, which means that they implicitly assume that $f(\alpha)$ is always concave. It must be observed that some of the reported spectra appear to be affine (see, e.g., Fig. 2 in Ref. [32]), so they are not problematic - they satisfy the concavity assumption. However, some other spectra are clearly nonconcave; see, e.g., Fig. 1 in Ref. [32] and Figs. 2 and 3 in Ref. [34]. For these, we must be careful because the observed nonconcavity could be a finite-size effect inherent to the fact that $f(\alpha)$ is computed numerically for a finite coarse-graining resolution $\varepsilon$. Thus it could be that the nonconcavity of $f(\alpha)$ observed for $\varepsilon>0$ disappears as $\varepsilon \rightarrow 0$. To verify this, one would need to perform a finite-size analysis of the data by computing $f(\alpha)$ for decreasing values of $\varepsilon$ and study the convergence of the results. Similar finite-size analyses have been performed in the context of numerical calculations of the microcanonical entropy function; see Refs. [35, 36, 37].

\section{Spectrum of dynamical indices}

First-order phase transitions have also been studied in the context of chaotic systems at the level of an entropylike quantity referred to as the spectrum of dynamical indices or expansion-rate spectrum [2, 33, 38, 39]. These phase transitions typically occur for nonhyperbolic dynamical systems.

It would take us too far to explain the notion of dynamical indices and the many examples for which this quantity has been studied. Let us only mention that the spectrum of dynamical indices is a dynamical analog of $f(\alpha)$, and that affine and nonconcave spectra of dynamical indices have been reported in the literature; see, e.g., Refs. [33, 39, 40, 41, 42, 43]. Most of these references, unfortunately, share the same problem as those discussed so far: they assume that the spectrum of dynamical indices can always be calculated as the LF transform of a dynamical analogue of the free energy function $\tau(q)$. Most of them assume this even when reporting the computation of nonconcave spectra; see Yoshida and Miyazaki [43] for a noticeable exception.

\section{GENERALIZED FREE ENERGY FUNCTIONS}

At this point, we have emphasized more than once that a nonconcave multifractal spectrum cannot be calculated as the LF transform of its corresponding free energy function. Our goal in this section is to offer a practical solution to this problem by illustrating a method for obtaining nonconcave spectra through LF transforms of a generalized form of free energy function. The method was proposed recently in Refs. 20, 44] in the context of nonconcave entropies within the microcanonical ensemble, and will be illustrated here in the context of the first example considered in Sec. II.

The form of generalized free energy that we shall consider is based on a generalization of the partition function given by

$$
Z_{\varepsilon}(q, g)=\sum_{i} \varepsilon^{q \alpha_{i}+g\left(\alpha_{i}\right)}
$$

where $\alpha_{i}$ represents the local fractal exponent associated with the probability $p_{\varepsilon, i}$, and $g$ is an arbitrary smooth function. This new form generalizes the standard canonical partition function, in the sense that

$$
Z_{\varepsilon}(q, g=0)=Z_{\varepsilon}(q)=\sum_{i} \varepsilon^{q \alpha_{i}}
$$

For definiteness, we shall adopt the choice $g(\alpha)=\gamma \alpha^{2}$ with $\gamma \in \mathbb{R}$. Therefore, the generalized partition function that we consider is

$$
Z_{\varepsilon}(q, \gamma)=\sum_{i} \varepsilon^{q \alpha_{i}+\gamma \alpha_{i}^{2}}
$$

We call this partition function the Gaussian partition function; its corresponding free energy function

$$
\tau(q, \gamma)=\lim _{\varepsilon \rightarrow 0} \frac{\ln Z_{\varepsilon}(q, \gamma)}{\ln \varepsilon}
$$

is called the Gaussian free energy. Note that this new free energy is a function of two real parameters, $q$ and $\gamma$, and that $\tau(q, \gamma=0)=\tau(q)$.

The rationale for generalizing the standard free energy function $\tau(q)$ to $\tau(q, \gamma)$ is that it modifies the structure of the LF transform which connects $\tau(q)$ with $f(\alpha)$, and thus modifies the conditions which ensure that $f(\alpha)$ can be written as the LF transform of a free energy function. We spare the reader with the details of this modification which can be found in Refs. 20, 44]. For our purpose, we shall only note the generalized versions of the LF transforms that connect $\tau(q, \gamma)$ and $f(\alpha)$; they are given by

$$
\tau(q, \gamma)=\inf _{\alpha}\left\{q \alpha+\gamma \alpha^{2}-f(\alpha)\right\}
$$

and

$$
f(\alpha)=\inf _{q, \gamma}\left\{q \alpha+\gamma \alpha^{2}-\tau(q, \gamma)\right\} .
$$

The first LF transform holds, like its standard version $(\gamma=0)$, for any spectrum $f(\alpha)$, be it concave or not. The surprising virtue of the second LF transform is that it also holds true for basically any $f(\alpha)$, contrary to the standard version $(\gamma=0)$ which applies only when $f(\alpha)$ is concave. Rather than proving this result, we shall verify that it is valid for the nonconcave multifractal spectrum 
shown in Fig. 1(a). That is, we shall obtain that nonconcave spectrum by inverting, in the manner of Eq.(28), its associated Gaussian free energy $\tau(q, \gamma)$.

First, we calculate $\tau(q, \gamma)$ starting from $Z_{\varepsilon}(q, \gamma)$ :

$$
Z_{\varepsilon}(q, \gamma)=\sum_{i} \varepsilon^{q \alpha_{i}+\gamma \alpha_{i}^{2}} \sim \varepsilon^{q / 2+\gamma / 4}+\varepsilon^{-1} \varepsilon^{q+\gamma} .
$$

Taking the limit $\varepsilon \rightarrow 0$ yields

$$
\tau(q, \gamma)=\min \{q / 2+\gamma / 4, q+\gamma-1\}
$$

The solution of the minimum can be found explicitly; it has the form

$$
\tau(q, \gamma)= \begin{cases}q / 2+\gamma / 4 & q \geq q_{\gamma} \\ q+\gamma-1 & q<q_{\gamma}\end{cases}
$$

where $q_{\gamma}=-3 \gamma / 2+2$. Next, we apply formula (28) using this solution for $\tau(q, \gamma)$. This leads us to solving the following variational problem:

$$
I=\inf _{q, \gamma}\left\{\begin{array}{ll}
q \alpha+\gamma \alpha^{2}-q / 2-\gamma / 4 & q \geq q_{\gamma} \\
q \alpha+\gamma \alpha^{2}-q-\gamma+1 & q<q_{\gamma}
\end{array}\right\} .
$$

Grouping the variables together, this is equivalent to

$$
I=\inf _{q, \gamma}\left\{\begin{array}{ll}
q(\alpha-1 / 2)+\gamma\left(\alpha^{2}-1 / 4\right) & q \geq q_{\gamma} \\
q(\alpha-1)+\gamma\left(\alpha^{2}-1\right)+1 & q<q_{\gamma}
\end{array}\right\} .
$$

Let $f_{1}(\alpha, q, \gamma)$ denote the top expression in the brackets and $f_{2}(\alpha, q, \gamma)$ the lower one. With this notation, it can be noted that, for $\alpha=1 / 2$,

$$
f_{2}(1 / 2, q, \gamma)>f_{1}(1 / 2, q, \gamma)=0
$$

for all $q<q_{\gamma}$ and $\gamma \in \mathbb{R}$. Therefore,

$$
\inf _{q<q_{\gamma}, \gamma} f_{2}(\alpha, q, \gamma)=f_{1}=0
$$

and $I=0$ at $\alpha=1 / 2$. Similarly, for $\alpha=1$, we have

$$
f_{1}(1, q, \gamma) \geq f_{2}(1, q, \gamma)=1
$$

for all $q \geq q_{\gamma}$ and $\gamma \in \mathbb{R}$, so that $I=f_{2}=1$ at $\alpha=1$. For all other values of $\alpha$, it is possible to set $q$ and $\gamma$ in such a way that $I=-\infty$. At the end, we are left with

$$
I= \begin{cases}0 & \alpha=1 / 2 \\ 1 & \alpha=1 \\ -\infty & \text { otherwise }\end{cases}
$$

which is the precise expression of $f(\alpha)$, as given in Eq. (6). Therefore, we have shown that this nonconcave spectrum can be expressed as in Eq.(28) as a modified LF transform of a generalized free energy.

The same method can be applied to calculate other nonconcave spectra. In fact, it has been conjectured that the method can be used to calculate any nonconcave spectrum (viz., nonconcave entropy function) as the LF transform of a properly-chosen generalized free energy. More details about this universality property can be found in Refs. [20, 44].

\section{CONCLUSION}

We have shown in this paper that one must be careful when calculating the singularity spectrum of multifractals as the LF transform of its corresponding free energy, since LF transforms can only yield concave functions. This word of caution has implications for most of the studies published so far on multifractals, including those on multifractal models of turbulence, as they have taken for granted that the multifractal spectrum is the LF transform of the free energy no matter what the spectrum looks like. This, as we have seen, is only true if the spectrum is concave; if it is nonconcave, then one must resort to calculate it directly from its definition. Another possibility is to use a generalization of the canonical ensemble which can be used to extract nonconcave entropies from a generalized version of the free energy function. This way of doing was sketched here in the context of a simple example of nonconcave multifractal spectrum, and is presented in full details in Refs. [20, 44].

In the end, it should be noted that the results that we have discussed in this paper are not special to multifractals, but apply actually to any field of investigation which uses LF transforms. In statistical mechanics, for example, the LF transform that connects the entropy function of the microcanonical ensemble with the free energy function of the canonical ensemble becomes noninvolutive when the entropy is nonconcave. When this happens, we say that there is nonequivalence of ensembles [45, 46], since one is then unable to obtain the true entropy function of the microcanonical ensemble solely from the knowledge of the free energy of the canonical ensemble. The notion of generalized canonical ensemble has been developed precisely in this context.

Similarly, in large deviation theory, it has been known for some time that nonconvex rate functions cannot be calculated by means of LF transforms of functions analogous to free energy functions [47, 48, 49, 50]. A multifractal spectrum is in essence an entropy function, and an entropy function is in essence a rate function [51, 52, 53, 54], so there is actually a deep connection with what we have presented here and what is known in large deviation theory [50]. For example, the result relating the differentiability of $\tau(q)$ and the (strict) concavity of $f(\alpha)$ can be put in correspondence with a result of large deviation theory known as the Gärtner-Ellis Theorem [19, 49, 55]. Furthermore, the result stating that the LF transform of $f(\alpha)$ always yields $\tau(q)$ (see Sec. III) can be put in correspondence with a large deviation result known as Varadhan Theorem; see Refs. [19, 55].

From these correspondences, it is but a small step to conjecture that nonconcave entropies or, more generally, nonconvex rate functions should show up in other physical theories in which large deviations are at play. One such theory that comes to mind is the thermodynamic formalism of dynamical systems [2, 56]; another is nonequilibrium statistical mechanics. We have already alluded to the first theory when discussing the spectrum 
of dynamical indices in Sec. IV. Concerning nonequilibrium statistical mechanics, the reader will find an example of nonconvex rate function in a recent paper by Imparato and Peliti [57] (see their Fig. 12). This paper discusses thermodynamic fluctuations in systems driven out of equilibrium. A large deviation result in that context is referred to as a fluctuation theorem, whereas a rate function is called a fluctuation function.

\section{Addendum}

Since this paper was submitted for publication, we became aware of two recent papers by Testud [58, 59] and one by Riedi [60] in which examples of nonconcave multifractal spectra are discussed.

\section{Acknowledgments}

H.T. was supported by NSERC (Canada), the Royal Society of London, and FCAR (Québec) during the initial phase of this work. C.B. is supported by a Springboard Fellowship from EPSRC (UK).
* Electronic address: htouchet@alum.mit.edu

$\dagger$ Electronic address: c.beck@qmul.ac.uk

[1] K. Falconer, Fractal Geometry: Mathematical Foundations and Applications (Wiley, New York, 1990).

[2] C. Beck and F. Schlögl, Thermodynamics of Chaotic Systems (Cambridge University Press, Cambridge, 1993).

[3] B. B. Mandelbrot, Multifractals and $1 / f$ Noise (Springer, New York, 1999).

[4] U. Frisch and G. Parisi, in Turbulence and Predictibility of Geophysical Flows and Climate Dynamics, edited by M. Ghil, R. Benzi, and G. Parisi (North-Holland, Amsterdam, 1985).

[5] T. C. Halsey, M. H. Jensen, L. P. Kadanoff, I. Procaccia, and B. I. Shraiman, Phys. Rev. A 33, 1141 (1986).

[6] R. Benzi, G. Paladin, G. Parisi, and A. Vulpiani, J. Phys. A 17, 3521 (1984).

[7] R. Benzi, L. Biferale, G. Paladin, A. Vulpiani, and M. Vergassola, Phys. Rev. Lett. 67, 2299 (1991).

[8] R. Benzi, S. Ciliberto, R. Tripiccione, C. Baudet, F. Massaioli, and S. Succi, Phys. Rev. E 48, 29 (1993).

[9] G. Stolovitzky, K. R. Sreenivasan, and A. Juneja, Phys. Rev. E 48, 3212 (1993).

[10] D. Schertzer and S. Lovejoy, Phys. Chem. Hydrodyn. J. 6, 623 (1985).

[11] D. Schertzer, S. Lovejoy, F. Schmitt, Y. Chigirinskaya, and D. Marsan, Fractals 5, 427 (1997).

[12] D. Schertzer and S. Lovejoy, J. Geophys. Res. 92, 9693 (1987).

[13] D. Schertzer and S. Lovejoy, in Fractals: Physical Origin and Consequences, edited by L. Pietronero (Plenum, New York, 1989), p. 49.

[14] D. Schertzer and S. Lovejoy, eds., Scaling, Fractals and Non-Linear Variability in Geophysics (Kluwer, Boston, 1991).

[15] F. Schmitt, D. Schertzer, and S. Lovejoy, Appl. Stochastic Models Data Anal. 15, 29 (1999).

[16] G. Paladin and A. Vulpiani, Phys. Rep. 156, 147 (1987).

[17] E. Ott, W. D. Withers, and J. A. Yorke, J. Phys. Stat. 36, 687 (1984).

[18] R. T. Rockafellar, Convex Analysis (Princeton University Press, Princeton, 1970).

[19] R. S. Ellis, Entropy, Large Deviations, and Statistical Mechanics (Springer-Verlag, New York, 1985).

[20] M. Costeniuc, R. S. Ellis, H. Touchette, and B. Turking- ton, J. Stat. Phys. 119, 1283 (2005), cond-mat/0408681.

[21] K. Huang, Statistical Mechanics (Wiley, New York, 1987).

[22] R. S. Ellis, H. Touchette, and B. Turkington, Physica A 335, 518 (2004).

[23] A.-M. S. Tremblay, Phys. Lett. A 116, 329 (1986).

[24] B. Fourcade and A.-M. S. Tremblay, Phys. Rev. A 36, 2352 (1987).

[25] P. Szépfalusy, T. Tél, A. Csordás, and Z. Kovas, Phys. Rev. A 36, 3525 (1987).

[26] A. Csordás and P. Szépfalusy, Phys. Rev. A 39, 4767 (1989).

[27] M. H. Jensen, in Universalities in Condensed Matter, edited by R. Jullien, L. Peliti, R. Rammal, and N. Boccara (Springer, Heidelberg, 1988), pp. 233-235.

[28] H. Xu, N. Ouellette, and E. Bodenschatz, Phys. Rev. Lett. 96, 114503 (2006).

[29] T. A. Witten and L. M. Sander, Phys. Rev. Lett. 47, 1400 (1981).

[30] T. C. Halsey, P. Meakin, and I. Procaccia, Phys. Rev. Lett. 56, 854 (1986).

[31] M. H. Jensen, A. Levermann, J. Mathiesen, and I. Procaccia, Phys. Rev. E 65, 046109 (2002).

[32] H. Hata, T. Horita, H. Mori, T. Morita, and K. Tomita, Prog. Theoret. Phys. 81, 11 (1989).

[33] H. Mori, H. Hata, T. Horita, and T. Kobayashi, Prog. Theoret. Phys. Suppl. 99, 1 (1989).

[34] H. Tominaga, H. Hata, T. Horita, H. Mori, and K. Tomita, Prog. Theoret. Phys. 84, 18 (1990).

[35] M. Kastner, Ph.D. thesis, Universität ErlangenNürnberg (2000).

[36] M. Pleimling, H. Behringer, and A. Hüller, Phys. Lett. A 328, 432 (2004).

[37] H. Behringer, M. Pleimling, and A. Hüller, J. Phys. A 38, 973 (2005).

[38] M. Sano, S. Sato, and Y. Sawada, Prog. Theoret. Phys. 76, 945 (1986).

[39] H. Hata, T. Horita, H. Mori, T. Morita, and K. Tomita, Prog. Theoret. Phys. 80, 809 (1988).

[40] T. Horita, H. Hata, H. Mori, T. Morita, K. Tomita, S. Kuroki, and H. Okamoto, Prog. Theoret. Phys. 80, 793 (1988).

[41] K. Tomita, H. Hata, T. Horita, H. Mori, and T. Morita, Prog. Theoret. Phys. 80, 953 (1988). 
[42] K. Tomita, H. Hata, T. Horita, H. Mori, T. Morita, H. Okamoto, and H. Tominaga, Prog. Theoret. Phys. 81, 1124 (1989).

[43] T. Yoshida and S. Miyazaki, Prog. Theoret. Phys. Suppl. 99, 64 (1989).

[44] M. Costeniuc, R. S. Ellis, H. Touchette, and B. Turkington, Phys. Rev. E 73, 026105 (2006).

[45] R. S. Ellis, K. Haven, and B. Turkington, J. Stat. Phys. 101, 999 (2000).

[46] H. Touchette, R. S. Ellis, and B. Turkington, Physica A 340, 138 (2004).

[47] I. H. Dinwoodie and S. L. Zabell, Ann. Prob. 20, 1147 (1992).

[48] D. Ioffe, Stat. Prob. Lett. 18, 297 (1993).

[49] R. S. Ellis, Scand. Actuarial J. 1, 97 (1995).

[50] H. Touchette, Ph.D. thesis, McGill University (2003).
[51] D. Plachky and J. Steinebach, Per. Math. Hung. 6, 343 (1975).

[52] O. E. Lanford, in Statistical Mechanics and Mathematical Problems, edited by A. Lenard (Springer, Berlin, 1973), vol. 20 of Lecture Notes in Physics, pp. 1-113.

[53] G. Zohar, Stoc. Proc. Appl. 79, 229 (1999).

[54] D. Veneziano, Fractals 10, 117 (2002).

[55] Y. Oono, Prog. Theoret. Phys. Suppl. 99, 165 (1989).

[56] D. Ruelle, Thermodynamic Formalism (Cambridge University Press, Cambridge, 2004), 2nd ed.

[57] A. Imparato and L. Peliti, Phys. Rev. E 72, 046114 (2005).

[58] B. Testud, C. R. Acad. Sci. Paris, Ser. I 340, 653 (2005).

[59] B. Testud, Nonlinearity 19, 1201 (2006).

[60] R. Riedi, J. Math. Anal. Appl. 189, 462 (1995). 